\title{
Simultaneous transarterial chemoembolization combined with radiofrequency ablation for treatment of large hepatocellular carcinoma: 3 years follow-up
}

\author{
Duan Feng ${ }^{1}$, Bai Yan-Hua ${ }^{1}$, Cui Li ${ }^{1}$, Li Xiao-Hui ${ }^{1}$ and Yan Jie-Yu ${ }^{1}$ \\ ${ }^{1}$ Department of Interventional Radiology, The General Hospital of Chinese People's Liberation Army, Beijing 100853, China \\ Correspondence to: Duan Feng, email: duanfeng@vip.sina.com
}

Keywords: chemoembolization; radiofrequency; hepatocellular carcinoma; simultaneous combination

Received: August 21, $2017 \quad$ Accepted: November 16, $2017 \quad$ Published: January 02, 2018

Copyright: Feng et al. This is an open-access article distributed under the terms of the Creative Commons Attribution License 3.0 (CC BY 3.0), which permits unrestricted use, distribution, and reproduction in any medium, provided the original author and source are credited.

\section{ABSTRACT}

Aims: To evaluate the recurrence and mid-term survival in patients with large hepatocellular carcinoma (HCC, diameter $\geq 8 \mathrm{~cm}$ ) after simultaneous transarterial chemoembolization (TACE) combined with radiofrequency ablation (RFA).

Materials and Methods: From March 2010 to Nov 2013, 46 consecutive patients of HCC with large HCC $(8.17-8.80 \mathrm{~cm} ; 8.40 \pm 2.62 \mathrm{~cm})$ were treated by simultaneous TACE combined with RFA, with single tumor in $\mathbf{3 5}$ of $\mathbf{4 6}$ patients. Progressive free survival (PFS) and overall survival (OS) were retrospectively analyzed.

Results: 46 sessions of simultaneous TACE combined with RFA were performed successfully without significant complications. The PFS and OS median survival time were $9.40 \pm 1.31$ or $18.43 \pm 1.34$ months at 2-year, $10.21 \pm 1.58$ or $26.44 \pm$ 2.26 months at 3-year, respectively. Patient survival rates were $67.50 \%$ at 2 year and $55.67 \%$ at 3years, respectively. Of patients with progression, comprehensive treatment including TACE and sorafenib was performed.

Conclusions: Simultaneous TACE combined with RFA is a safe and effective treatment modality in patients with large HCCs, the preliminary data make it a promising treatment modality.

\section{INTRODUCTION}

Hepatocellular carcinoma (HCC) is one of the most common cancers and leading causes of tumor-related deaths $[1,2]$. Large HCC patients (tumor larger than 8 $\mathrm{cm}$ ) are generally at the advanced stage of the disease and with poor prognosis $[3,4]$. Hepatic resection may be the curative treatment for patients suffering from single large HCC with well-preserved hepatic function (Child-Pugh A). However, low proportions of patients are suitable, and the incidence of postoperative recurrence is still high [5].

Transcatheter arterial chemoembolisation (TACE) and radiofrequency ablation (RFA) are often used for unresectable HCC treatment. The best candidates for TACE are asymptomatic patients with well-preserved liver function and a solitary or limited multifocal HCC without vascular invasion or extrahepatic spread. However, TACE alone results in incomplete tumor necrosis. Although the therapeutic efficacy of RFA of small HCC (i.e., $<3 \mathrm{~cm}$ ) could be comparable to surgical operation $[6,7]$, RFA has shown poor local tumor control when the tumor size exceeds $3 \mathrm{~cm}[8,9]$. Therefore, the combination of TACE with radiofrequency ablation (RFA) may enhance efficacy against large $\mathrm{HCC}$ and prolong survival time in patients.

In the presence work, we aim to reveal the efficacy of combination of TACE immediately with RFA in the treatment of large HCC. We retrospectively summarized and followed up 46 patients treated between March 2010 and Nov 2013 to assess the mid-term efficacy of TACE combined with simultaneously RFA as a novel strategy of large HCC treatment.

\section{MATERIALS AND METHODS}

A total of 46 patients, that were identified consecutively, with large HCC (at least 1 lesion's diameter $\geq 8 \mathrm{~cm}$ ) were included in this study. Baseline clinical characteristics of HCC patients are shown in Table 1 and Table 2, (1) patients included 42 males and 4 females; (2) median age of patients was 53.5 (36-70) years; (3) patients diagnosed as advanced- 
stage HCC according to the BCLC staging classification (42/4; B/C) with Child-Pugh class A (45/46) or Child-Pugh B (1/46) liver function. The mean maximum tumor size was 8.17 $(1.2-14.0) \mathrm{cm}$. Sex, age, tumor stage, size of tumor, number of tumor, child-pugh score, pseudocapsule and vascular invasion (tumor thrombus in the first branch or trunk of the portal vein) were used as subgroup factors for subgroup analysis. The study protocol was approved by the Ethics Committee of the Hospital, and received the informed consent from patients. The diagnoses of HCC patients were based on imaging findings and/or AFP levels. Tumor stage was identified according to the Barcelona Clinic Liver Cancer (BCLC) staging classification system. Patients were surgically unsuitable, and without arterio-venous fistula, or ascites.

\section{Treatment protocol}

After a routine preoperative preparation, TACE was performed first under sterile conditions, with the patient under general anesthesia [10]. The right femoral artery was cannulated using a 4F vascular sheath (Radifocus Introducer II; Terumo Corp., Japan) by Seldinger's technique. Selective angiography of the celiac artery, superior mesenteric artery was performed using a $4 \mathrm{~F}$ hepatic artery catheter (HA, Terumo Corp., Japan) inserted through the vascular sheath. Maximum catheter selectivity of the hepatic artery was achieved using a microcatheter (Progreat, Terumo Corp., Japan), with administration of embolic agent from the feeding arteries of the tumor lesion. Drug dosages per procedure varied, ranging from 15-20 mL for lipiodol (Guerbet Corp., France), 30-50 mg of doxorubicin (Pfizer Pharmaceuticals Ltd, USA), 100-150 mg oxilaplatin (Sanofi Pharmaceuticals Co., Ltd, France), depending on the size of the tumors and laboratory results. Lipiodol-chemotherapeutic agents were administered until stasis, minimizing reflux into non-target vessels. Injection was continued until near stasis and was observed in the artery that directly fed the tumor (i.e., the contrast column - cleared within 2-5 heartbeats). Gelatin sponge was injected as supplement if stasis was not achieved after $20 \mathrm{ml}$ lipiodol has been delivered. Collateral artery embolization was performed if branches, such as inferior phrenic artery, internal thoracic artery and omental branches etc, feed the tumor.

Percutaneous RFA was immediately performed after TACE under general anesthesia with guidance of DSA combined with Dyna-CT [11]. A multipolar RF probe (RITA company, cristal lake, IL, United States) with maximum ablation diameter of 5 or $7 \mathrm{~cm}$ and length of 10 or $15 \mathrm{~cm}$ was used in all cases. RF probe was inserted toward the tumor, with stain guided by fluoroscopy. Lateral view and postural view were used during puncture procedure. Then dyna-CT was performed to confirm the position of RF probe (Figure 1). Ablation started once target position were reached. The following settings were used: power: 150 to $200 \mathrm{~W}$; ablation time: $15 \mathrm{mins}$ after a temperature of $105^{\circ} \mathrm{C}$ were reached. RFA was performed two to five times according to the tumor size and maximum ablation diameter. Puncture tract ablation was performed to avoid bleeding and tumor seeding.

\section{Follow-up protocol and data collection}

Patients were followed up with repeat enhanced MRI every 1-2 months during the first year and then every 2-4 months afterwards. Local tumor recurrence, new tumor occurrence and metastasis were all considered progression. Of patients with progression, comprehensive treatment including TACE and sorafenib was performed. Two blinded authors performed over-reads of all clinical data interpretations and follow-up work.

\section{Statistical analysis}

A commercial statistical software package (SPSS for Windows, version 22.0; SPSS Inc, Chicago, Ill, USA) was used for data analysis. The estimated local tumor progression and overall survival rates were evaluated by the Kaplan-Meier method. A univariate Cox proportional hazard model was fitted to each variable. $P$ values $<0.05$ were considered as statistically significant.

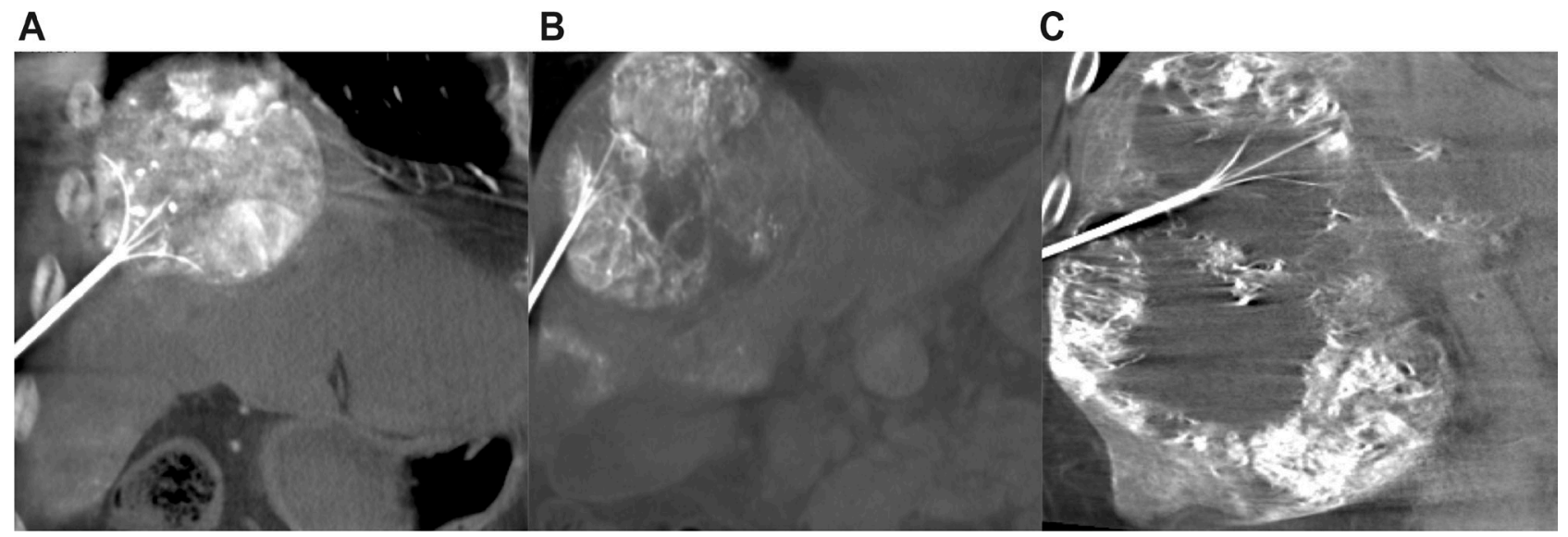

Figure 1: Dyna-CT image confirmed the position of RF probe; Inserted RF probe with an angle to avoid lung damage (A-C). 
Table 1: Baseline characters of patients before treatment

\begin{tabular}{ll}
\hline Characters & Before treatment \\
\hline Sex, M/F & $42 / 4$ \\
Age, years, median (range) & $53.5(36-70)$ \\
BCLC stage B/C ${ }^{\dagger}$ & $42 / 4$ \\
Child-Pugh status A/B/C & $45 / 1 / 0$ \\
ECOG performance status 0/1 ${ }^{\dagger \dagger}$ & $42 / 4 / 0$ \\
Laboratory values, median (range) & \\
WBC count, $10^{9} / \mathrm{L}$ & $4.98(2.23-10.09)$ \\
Platelet count, $10^{9} / \mathrm{L}$ & $158(49-371)$ \\
Hemoglobin, g/dL & $135(76-159)$ \\
Serum AST, IU/L & $18.85(16.20-101.60)$ \\
Serum ALT, IU/L & $28.7(9.6-178.8)$ \\
Serum total bilirubin, mg/dL & $13.2(5.0-41.4)$ \\
Serum albumin, g/dL & $38.45(28.8-45.1)$ \\
INR & $1.10(0.92-1.33)$ \\
Serum creatinine, mg/dL & $65.05(40.30-106.20)$ \\
Serum alpha-fetoprotein, ng/mL & $7.86(1.28-24200.00)$ \\
Serum alpha-fetoprotein, baseline $>20$ & $170.80(20.02-24200.00)(n=17)$ \\
Tumor burden and distribution & \\
Unifocal/multifocal & $35 / 11$ \\
Unilobar/bilobar & $44 / 2$ \\
lesions diameter (cm) & \\
Largest lesion diameter, median, mean, range & $14.0,8.40,8.17,1.2-14.0$ \\
\hline Barcel Clinic Liver Can &
\end{tabular}

Barcelona Clinic Liver Cancer staging system

${ }^{\dagger}$ Eastern Cooperative Oncology Group performance status.

\section{Table 2: Baseline characters of patients after treatment}

\begin{tabular}{ll}
\hline Characters & After treatment \\
\hline Laboratory values, median (range) & \\
WBC count, $10^{9} / \mathrm{L}$ & $4.985(2.300-9.050)$ \\
Platelet count, $10^{9} / \mathrm{L}$ & $141(44-259)$ \\
Hemoglobin, g/dL & $136.5(98.0-165.0)$ \\
Serum AST, IU/L & $28.2(15.0-103.7)$ \\
Serum ALT, IU/L & $32.15(8.40-74.30)$ \\
Serum total bilirubin, mg/dL & $14.45(5.40-44.80)$ \\
Serum albumin, g/dL & $37.75(24.30-46.30)$ \\
INR & $1.08(0.95-1.41)$ \\
Serum creatinine, $\mathrm{mg} / \mathrm{dL}$ & $65.7(45.4-134.6)$ \\
Serum alpha-fetoprotein, ng/mL & $5.60(1.12-24200.00)$ \\
Serum alpha-fetoprotein, baseline $>20$ & $916.2(21.1-24200.0)(n=17)$ \\
\hline
\end{tabular}

\section{RESULTS}

\section{Treatment response}

Next, the response of patients to combination of therapies was determined. Figure 2 showed a typical case response to combination therapy. As shown in the table, there is no significant difference of lab examination between, before and after treatment. The 2-year and 3-year OS of patients are $18.43 \pm 1.34$ and $26.44 \pm 2.26$ months (mean $\pm \mathrm{sd}$ ), respectively. The 2-year and 3-year PFS of patients are $9.40 \pm 1.31$ and $10.21 \pm 1.58($ mean $\pm \mathrm{sd})$, 
respectively. The OS and PFS curves were shown in Figure 3.

Subset group analysis revealed similar OS and PFS in most sub-groups (Table 3 ). The only differences were obtained between vascular invasion/non-vascular invasion group and male/female group. The OS of vascular invasion group and female group were significantly shorter than non-vascular invasion group $(p=0.019)$ and male group $(p=0.031)$.

\section{Side effects and complications}

Clinical adverse events included fever, pain, nausea, fatigue, transient reduction of blood counts and transient elevation of AST and ALT level, but were mostly limited to grade 1 and 2 (Table 2). No complications associated with our procedure were noted.

\section{DISCUSSION}

Hepatocellular carcinoma (HCC) is one of the most fatal causes of liver-disease related death. Although significant progress has been made in clinical treatment in recent years, the clinical outcome or prognosis of large $\mathrm{HCC}$ is still poor.

The combined application of TACE with RFA was previously reported and has shown a synergistic effect on inactivation of HCC [12]. TACE plus RFA may enhance the efficacy of treatment, prolong patient survival and in turn decrease recurrence rate, so combination of TACE and RFA would be a hopeful choice for large HCCs those are unsuitable for surgical operation $[5,13]$. Currently, TACE-RFA combination therapies are often performed separately. The interval between TACE and RFA varies from 1 day to 4 weeks, during which recanalization after embolization, collateral formation and elimination of lipiodol-chemotherapeutic agents may occur. Therefore,
TACE or RFA often does not strictly concurrent, and the effects of TACE or RFA alone are not fully synergistic [6, $14,15]$. Therefore, it is valuable to reveal the efficacy of TACE combined RFA.

Presented work, a 3 years follow-up focusing on simultaneous TACE combined with RFA treating large hepatocellular carcinoma, the outcome seemed to be effective. This combination therapy may have several advantages: First, digital subtraction angiography (DSA) or Dyna CT could clearly visualize blood vessels. Therefore, DSA and dyna CT allow us to successful liver puncture and to intra-operatively verify the efficacy $[16,17]$. Second, in simultaneous combination therapy, lipiodol precipitated around the lesion has not been expurgated and may work as thermal energy transmitting medium to enhance the efficacy of ablation to inactivate the surrounding micro-environments of HCC [18]. This situation may improve efficacy of simultaneous RFA and limit recurrence. Third, TACE may block the blood flow in local tumor and in turn reduce the heat loss during simultaneous RFA [19]. Fourth, RFA immediately after TACE may also induce the injury of local lesion from liquefactive necrosis to coagulation necrosis and attenuate the side effects of TACE [20]. Finally, simultaneous combination of TACE and RFA could be performed within a single session, and in turn reduce the burden of patients. Generally, simultaneous combination of TACE and RFA may fully exert the synergistic effects of chemotherapeutics and thermal ablation and our study showed that there were no significant adverse effects. Therefore, simultaneous combination of TACE and RFA would be a valuable strategy to improve the survival and efficacy of large HCC treatment.

Our COX proportional hazard model multivariate analysis indicated that vascular invasion was a prognostic factor. This is mainly because the vascular invasion patients had higher incidence of metastasis both intra-hepatic and

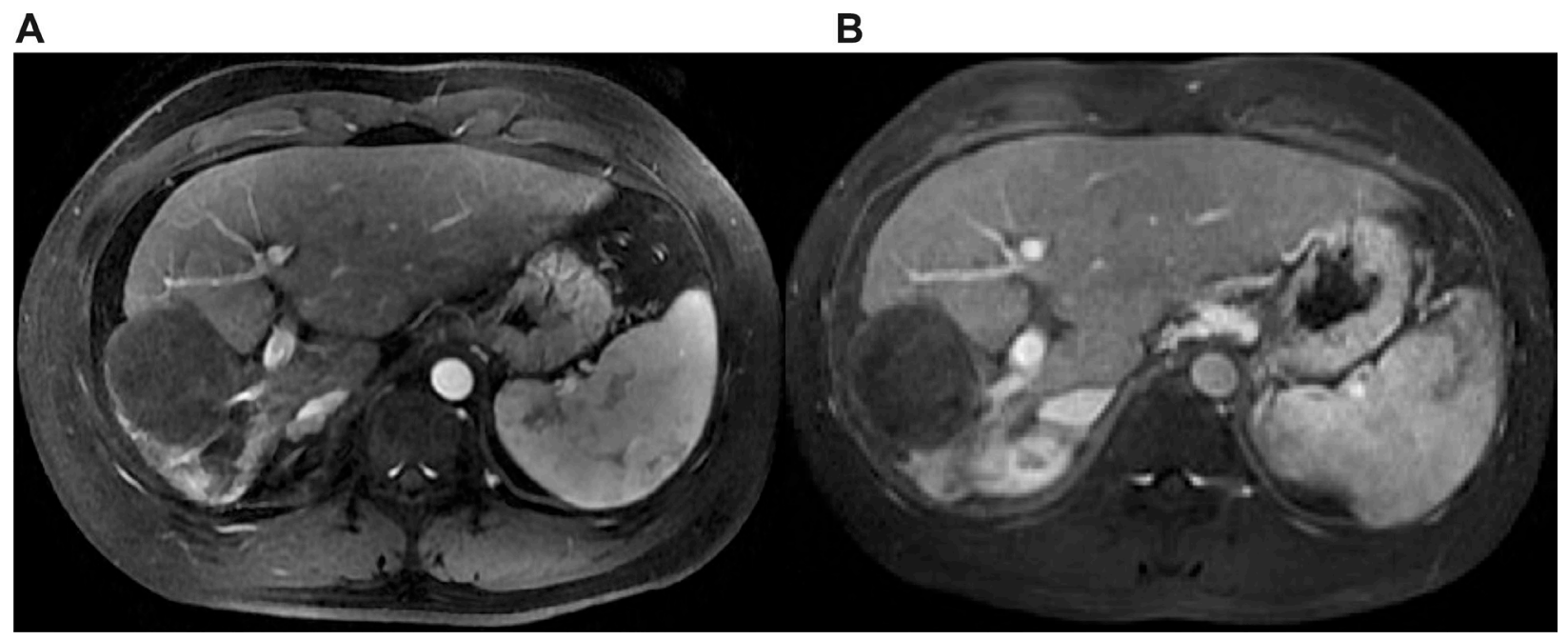

Figure 2: A 41 years old male patient re-examined 2 years and 4 years after combination therapy. (A) The 2-year MRI scan. (B) The 4-years MRI scan. Results indicate that HCC lesion was pyknosis and necrosis. 
Table 3: Sub-set group analysis

\begin{tabular}{|c|c|c|c|c|c|c|}
\hline Characters & $n$ & mOS (months) & 1 year survival & 2 year survival & 3 year survival & $p$ value \\
\hline Sex & & & & & & .031 \\
\hline Female & 4 & 10.5 & $2(0.5)$ & $1(0.25)$ & 0 & \\
\hline Male & 42 & 34.5 & $31(0.738)$ & $27(0.643)$ & $23(0.545)$ & \\
\hline Age (years) & & & & & & .264 \\
\hline$\leq 60$ & 37 & 34 & $26(0.703)$ & $22(0.595)$ & $20(0.541)$ & \\
\hline$>60$ & 9 & 35 & $7(0.778)$ & $6(0.667)$ & $4(0.444)$ & \\
\hline BCLC staging & & & & & & 657 \\
\hline B & 10 & 37.5 & $8(0.8)$ & $8(0.8)$ & $7(0.7)$ & \\
\hline $\mathrm{C}$ & 36 & 30 & $25(0.694)$ & $20(0.556)$ & $17(0.472)$ & \\
\hline Size of Tumor $(\mathrm{cm})$ & & & & & & .300 \\
\hline $8-10$ & 23 & 34 & $18(0.783)$ & $14(0.609)$ & $12(0.514)$ & \\
\hline$>10$ & 23 & 26 & $15(0.652)$ & $14(0.609)$ & $12(0.522)$ & \\
\hline Number of Tumor & & & & & & .087 \\
\hline Single & 35 & 34 & $26(0.743)$ & $23(0.657)$ & $20(0.567)$ & \\
\hline Multiple & 11 & 19 & $7(0.636)$ & $5(0.545)$ & $4(0.364)$ & \\
\hline Child-Paugh & & & & & & .640 \\
\hline A & 45 & 34 & $32(0.778)$ & $28(0.622)$ & $24(0.530)$ & \\
\hline B & 1 & 19 & $1(1.0)$ & 0 & 0 & \\
\hline Psuedocapsule & & & & & & .289 \\
\hline Yes & 11 & 44 & $10(0.909)$ & $9(0.818)$ & $7(0.636)$ & \\
\hline No & 35 & 30 & $23(0.657)$ & $19(0.543)$ & $17(0.482)$ & \\
\hline Vascular Invasion & & & & & & .019 \\
\hline Yes & 4 & 8 & $1(0.25)$ & $1(0.25)$ & 0 & \\
\hline No & 42 & 34.5 & $32(0.762)$ & $27(0.643)$ & $24(0.568)$ & \\
\hline AFP positive & & & & & & .051 \\
\hline Yes & 17 & 14.5 & $9(0.529)$ & $6(0.353)$ & $6(0.353)$ & \\
\hline No & 29 & 35 & $24(0.793)$ & $22(0.759)$ & $18(0.614)$ & \\
\hline
\end{tabular}
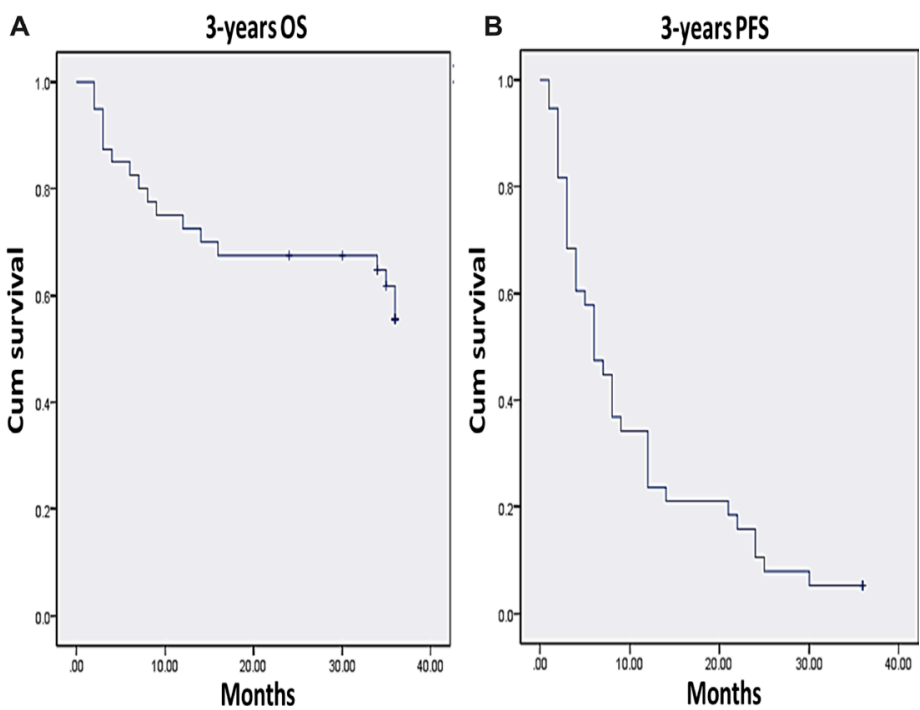

Figure 3: Kaplan meier univariate analysis of overall accumulative survival. Kaplan-Meier survival curves are shown for patients suffering from large HCC treated with combination therapy. (A) 3-year OS. (B) 3-year PFS. 
extra-hepatic than the non-vascular invasion group during our follow-up period. This indicated that the combination therapy had poor response with the tumor thrombus. Additionally, male/female group showed a statistically significant difference. However, this might be due to the fact that the number of female patients is too small to be evaluated. The pseudocapsule group showed better efficacy in clinical practice because of the possibility that the pseudocapsule could enhance the thermal aggregation effect of the ablation so as to better inactivate the tumor. It may be due to the limited number of cases, that the pseudocapsule group was not statistically significant. The other subgroups were not specific.

The shortcomings of this study include retrospective nature and the high loss rate. It is to conduct a prospective study of larger cohort with large HCC patients treated by TACE combined with simultaneous RFA to further assess the technical efficacy.

\section{Abbreviations}

hepatocellular carcinoma (HCC); transarterial chemoembolization (TACE); radiofrequency ablation (RFA); Progressive free survival (PFS); overall survival (OS); digital subtraction angiography (DSA); Barcelona Clinic Liver Cancer (BCLC); hepatic artery (HA).

\section{Author contributions}

DF, BYH and CL conceived and designed the study. DF and LXH performed interventional therapy. DF, BYH, CL, YJY and LXH wrote the paper. DF, BYH, CL and YJY reviewed and edited the manuscript. All authors read and approved the manuscript.

\section{ACKNOWLEDGMENTS}

First of all, I/we would like to extend my sincere gratitude to our departmental chair for all these support. I/ we am/are deeply grateful of the help from our physiciansengineers, nurses as well as other staff of the department.

\section{CONFLICTS OF INTEREST}

All authors have declared that no competing interests exist.

\section{FUNDING}

The authors received no specific funding for this work.

\section{REFERENCES}

1. European Association For The Study Of The Liver, European Organisation For Research And Treatment
Of Cancer. EASL-EORTC clinical practice guidelines: management of hepatocellular carcinoma. J Hepatol. 2012; 56:908-943.

2. Wang FS, Fan JG, Zhang Z, Gao B, Wang HY. The global burden of liver disease: the major impact of China. Hepatology. 2014; 60:2099-2108.

3. Alnaggar M, Niu L, Li J, Yao F, Wang Y, Zeng J, Ye J, Chen J, Mu F, Xu K. Cryoprotective therapy for large hepatocellular carcinoma: a study of 14 patients with a single lesion. Cryobiology. 2014; 69:457-61.

4. Xue TC, Jia QA, Ge NL, Chen Y, Zhang BH, Ye SL. Imbalance in systemic inflammation and immune response following transarterial chemoembolization potentially increases metastatic risk in large hepatocellular carcinoma. Tumour Biol. 2015; 36:8797-803.

5. Kim JW, Shin SS, Kim JK, Choi SK, Heo SH, Lim HS, Hur YH, Cho CK, Jeong YY, Kang HK. Radiofrequency ablation combined with transcatheter arterial chemoembolization for the treatment of single hepatocellular carcinoma of 2 to 5 $\mathrm{cm}$ in diameter: comparison with surgical resection. Korean J Radiol. 2013; 14:626-35.

6. Min JH, Lee MW, Cha DI, Jeon YH, Shin SW, Cho SK, Rhim H, Lim HK. Radiofrequency ablation combined with chemoembolization for intermediate-sized $(3-5 \mathrm{~cm})$ hepatocellular carcinomas under dual guidance of biplane fluoroscopy and ultrasonography. Korean J Radiol. 2013; $14: 248-58$.

7. Song MJ, Bae SH, Lee JS, Lee SW, Song do S, You CR, Choi JY, Yoon SK. Combination transarterial chemoembolization and radiofrequency ablation therapy for early hepatocellular carcinoma. Korean J Intern Med. 2016; 31:242-52.

8. Guo W, He X, Li Z, Li Y. Combination of Transarterial Chemoembolization (TACE) and Radiofrequency Ablation (RFA) vs. Surgical Resection (SR) on Survival Outcome of Early Hepatocellular Carcinoma: A Meta-Analysis. Hepatogastroenterology. 2015; 62:710-4.

9. Dai WC, Cheung TT, Chok KS, Chan AC, Sharr WW, Tsang SH, Yuen WK, Chan SC, Fan ST, Lo CM, Poon RT. Radiofrequency ablation versus transarterial chemoembolization for unresectable solitary hepatocellular carcinomas sized 5-8cm. HPB (Oxford). 2015; 17:226-31.

10. Feng D, Wei Y, Yan W. Trans-arterial chemoembolization and external beam radiation therapy for treatment of hepatocellular carcinoma with a tumor thrombus in the inferior vena cava and right atrium. Cancer Imaging. 2015, $15: 7$.

11. Wang ZJ, Wang MQ, Duan F. Transcatheter arterial chemoembolization followed by immediate radiofrequency ablation for large solitary hepatocellular carcinomas. World Journal of Gastroenterology, 2013, 19:4192-4199.

12. Ginsburg M, Zivin SP, Wroblewski K, Doshi T, Vasnani RJ, Van Ha TG. Comparison of Combination Therapies in the Management of Hepatocellular Carcinoma: Transarterial 
Chemoembolization with Radiofrequency Ablation versus Microwave Ablation. J Vasc Interv Radiol. 2015; 26:330-341.

13. Hou YF, Wei YG, Yang JY, Wen TF, Xu MQ, Yan LN, Li B. Combined hepatectomy and radiofrequency ablation versus TACE in improving survival of patients with unresectable BCLC stage B HCC. Hepatobiliary Pancreat Dis Int. 2016; 15:378-85.

14. Choe WH, Kim YJ, Park HS, Park SW, Kim JH, Kwon SY. Short-term interval combined chemoembolization and radiofrequency ablation for hepatocellular carcinoma. World J Gastroenterol. 2014; 20:12588-94.

15. Gadaleta C, Catino A, Ranieri G, Fazio V, GadaletaCaldarola G, Cramarossa A, Armenise F, Canniello E, Vinciarelli G, Laricchia G, Mattioli V. Single-step Therapy - Feasibility and Safety of Simultaneous Transarterial Chemoembolization and Radiofrequency Ablation for Hepatic Malignancies. In vivo. 2009; 23:813-820.

16. Shibata T, Shibata T, Maetani Y, Kubo T, Itoh K, Togashi $\mathrm{K}$. Transthoracic percutaneous radiofrequency ablation for liver tumors in the hepatic dome. J Vasc Interv Radiol 2004; 15:1323-1327.

17. Kato T, Yamagami T, Hirota T, Matsumoto T, Yoshimatsu R, Nishimura T. Transpulmonary radiofrequency ablation for hepatocellular carcinoma under real-time computed tomography-fluoroscopic guidance. Hepatogastroenterology 2008; 55:1450-1453.

18. Tamai T, Oshige A, Tabu K, Tabu E, Ijyuin S, Sakae H, Onishi H, Muromachi K, Saisyoji A, Oda K, Kumagai K, Mawatari S, Moriuchi A, et al. Utility of percutaneous radiofrequency ablation alone or combined with transarterial chemoembolization for early hepatocellular carcinoma. Oncol Lett. 2017; 14:3199-3206.

19. Bholee AK, Peng K, Zhou Z, Chen J, Xu L, Zhang Y, Chen M. Radiofrequency ablation combined with transarterial chemoembolization versus hepatectomy for patients with hepatocellular carcinoma within Milan criteria: a retrospective case-control study. Clin Transl Oncol. 2017; 19:844-852.

20. Chen L, Sun J, Yang X. Radiofrequency ablation-combined multimodel therapies for hepatocellular carcinoma: Current status. Cancer Lett. 2016; 370:78-84. 